\title{
Iteration of Differentiable
}

\section{Functions under $m$-Modal Maps with Aperiodic Kneading Sequences}

\author{
Maria de F. Correia, Carlos C. Ramos, and Sandra Vinagre
}

CIMA-UE and Department of Mathematics, University of Évora, Rua Romão Ramalho 59, 7000-671 Évora, Portugal

Correspondence should be addressed to Maria de Fátima Correia, mfac@uevora.pt

Received 30 March 2012; Accepted 21 May 2012

Academic Editor: Hans Engler

Copyright (C) 2012 Maria de Fátima Correia et al. This is an open access article distributed under the Creative Commons Attribution License, which permits unrestricted use, distribution, and reproduction in any medium, provided the original work is properly cited.

\begin{abstract}
We consider the dynamical system $(\mathcal{A}, T)$, where $\mathscr{A}$ is a class of differentiable functions defined on some interval and $T: \mathcal{A} \rightarrow \mathcal{A}$ is the operator $T \phi:=f \circ \phi$, where $f$ is a differentiable $m$-modal map. Using an algorithm, we obtained some numerical and symbolic results related to the frequencies of occurrence of critical values of the iterated functions when the kneading sequences of $f$ are aperiodic. Moreover, we analyze the evolution as well as the distribution of the aperiodic critical values of the iterated functions.
\end{abstract}

\section{Introduction}

In this paper, we pursue the study of the properties of certain infinite dynamical systems that arise from iterated differentiable interval maps. Regarding infinite dimensional systems, important progresses were obtained in the study of boundary value problems for partial differentiable equations reducible to difference equations, as those studied in [1-3]. An approach, in that context, using symbolic dynamics was made in [4-6].

We consider the dynamical system $(\mathcal{A}, T)$, where $\mathcal{A}$ is a class of differentiable real functions defined on some interval and $T: \mathcal{A} \rightarrow \mathcal{A}$ is the operator $T \phi:=f \circ \phi$, where $f$ is an $m$-modal map. The class $\mathcal{A}$ is the class of differentiable functions $\phi$ defined on an interval (eventually could be defined on $\mathbb{R}$ ) such that $\phi \in \mathscr{A}$ if $\phi \in C^{1}([0,1])$ with a finite number of critical points, satisfying the boundary conditions $\phi^{\prime}(0)=\phi^{\prime}(1)=0$ and $\operatorname{Im}(\phi) \subset I$, for a given interval $I$. The nature of $\mathcal{A}$ in terms of topological, metrical, or algebraic closure, for now, is not discussed since we are mainly interested in analyzing the qualitative changes of the elements in $\mathcal{A}$, under the iteration of $T$, from a combinatorial point of view. In particular, 
we study the changes on the number of critical points, the relative localization of the critical points and critical values. The developed techniques allow us to study, in future work, the changes, under iteration of $T$, of attributes of the elements in $\mathcal{A}$, such as the oscillations, the mean value, the measure, the mean derivative, the length of the graph, among others.

The dynamical system $(\mathcal{A}, T)$ has infinite dimension, although induced by a onedimensional discrete dynamical system $(I, f)$. From the topological point of view, the dynamical system $(I, f)$ is formally contained in $(\mathcal{A}, T)$, since the constant functions, $\phi \equiv c$, belong trivially to $\mathcal{A}$ and $T(c)=f(c)$. Moreover, a monotone function $\phi \in \mathcal{A}$ (nontrivial) determine a signed interval, $([\phi(0), \phi(1)],+)$ if $\phi$ is an increasing function and $([\phi(1), \phi(0)],-)$ if $\phi$ is a decreasing function. Then the dynamics of intervals under iteration of an interval map $f$ is also formally contained in $(\mathcal{A}, T)$.

We determine the itineraries of a sufficiently large number of critical values of the iterated functions using the algorithm introduced in [7]. We analyze the evolution and the distribution of these critical values whose frequencies are displayed in histograms. Moreover, we obtained a numerical result which relates the relative frequencies of critical values with the growth number of $f, s(f)$. In this paper, we analyze the case of $f$ being an $m$-modal map with aperiodic kneading sequences.

\section{Preliminaries on Symbolic Dynamics}

\subsection{Symbolic Dynamics for $m$-Modal Maps}

In this section, we describe some preliminaries on symbolic dynamics, in particular, aspects concerning to the $m$-modal maps on an interval $I$.

Let $I \subset \mathbb{R}$ be an interval. A map $f: I \rightarrow I$ is called m-modal if it is in $C^{1}(I)$ and has $m$ critical points. Let $c_{i}$, with $i=1,2, \ldots, m$, be the $m$ critical points of the map $f$ such that $c_{0}<c_{1}<\cdots<c_{m}<c_{m+1}$, where $c_{0}$ and $c_{m+1}$ represent the boundary points of the interval $I$. In these conditions, consider the partition of the interval $I$ into disjoint subsets

$$
I=I_{1} \cup I_{C_{1}} \cup I_{2} \cup I_{C_{2}} \cup \cdots \cup I_{C_{m}} \cup I_{m+1},
$$

where $I_{C_{i}}$ is the set $\left\{c_{i}\right\}, i=1,2, \ldots, m$, and $I_{i}, i=1,2, \ldots, m+1$, are the intervals

$$
I_{1}=\left[c_{0}, c_{1}\left[, \quad I_{2}=\right] c_{1}, c_{2}\left[, \ldots, \quad I_{m+1}=\right] c_{m}, c_{m+1}\right]
$$

A maximal interval on which $f$ is monotone is called a lap of $f$ and the number $l=l(f)$ of distinct laps is called the lap number of $f$. Thus, each interval $I_{i}, i=1,2, \ldots, m+1$, is a lap.

According to [8], the limit

$$
s(f)=\lim _{n \rightarrow \infty} l\left(f^{n}\right)^{1 / n}
$$

is a real number in the interval $[1, l(f)]$ and is called the growth number of $f$.

The topological entropy of $f$ is

$$
h_{\mathrm{top}}(f)=\log (s(f))=\lim _{n \rightarrow \infty} \frac{1}{n} l\left(f^{n}\right),
$$

see [9]. 
Next, to each point $x$ in $I_{i}, i=1,2, \ldots, m+1$, we assign the symbol $i, i=1,2, \ldots, m+1$, or $C_{i}, i=1,2, \ldots, m$, if $x=c_{i}, i=1,2, \ldots, m$. This assignment is called the address of $x$, and it is denoted by $\operatorname{ad}(x)$. The address of the point $x, \operatorname{ad}(x)$, is thus given by

$$
\operatorname{ad}(x)= \begin{cases}i & \text { if } x \in I_{i}, i=1,2, \ldots, m+1 \\ C_{i} & \text { if } x \in I_{C_{i}}, i=1,2, \ldots, m .\end{cases}
$$

As usual, we get a correspondence between orbits of points and symbolic sequences of the alphabet $\left\{1, C_{1}, 2, \ldots, m+1\right\}$, the itinerary of $x$ under $f$, which is defined by

$$
\operatorname{it}(x):=\operatorname{ad}(x) \operatorname{ad}(f(x)) \operatorname{ad}\left(f^{2}(x)\right) \cdots \in\left\{1, C_{1}, 2, \ldots, m+1\right\}^{\mathbb{N}}
$$

The orbits, under $f$, of the critical points are of special importance, in particular, their itineraries. Following [8], for each critical point the kneading sequence is $\mathcal{K}_{i}:=\operatorname{it}\left(f\left(c_{i}\right)\right), i=$ $1,2, \ldots, m$, and the collection of symbolic sequences $\mathcal{K}_{f}:=\left(\mathcal{K}_{1}, \ldots, \mathcal{K}_{m}\right)$ is called the $k n e a d i n g$ invariant of $f$.

A symbolic sequence $\left(i_{k}\right)_{k \geq 1}$ in $\left\{1, C_{1}, 2, \ldots, m+1\right\}^{\mathbb{N}_{0}}$ is called admissible, with respect to $f$, if it occurs as an itinerary for some point $x$ in $I$. The set of all admissible sequences in $\left\{1, C_{1}, 2, \ldots, m+1\right\}^{\mathbb{N}_{0}}$ is denoted by $\Sigma$.

In the sequence space $\Sigma$, we define the usual shift map $\sigma: \Sigma \rightarrow \Sigma$ by

$$
\sigma\left(i_{1} i_{2} i_{3} \cdots\right)=i_{2} i_{3} \cdots
$$

Moreover, the following relation with $f$ and the itinerary map is satisfied

$$
\sigma(\operatorname{it}(x))=\operatorname{it}(f(x))
$$

Therefore, we obtain the symbolic system $(\Sigma, \sigma)$ associated with the discrete dynamical system $(I, f)$.

An admissible word is a finite sub-sequence occurring in an admissible sequence. The set of admissible words of size $k$ occurring in some sequence from $\Sigma$ is denoted by $\boldsymbol{W}_{k}=\mathcal{W}_{k}(f)$. We define the cylinder set $I_{i_{0} i_{1} \cdots i_{k}} \subset I, i_{0} i_{1} \cdots i_{k} \in W_{k+1}$, by

$$
\begin{aligned}
I_{i_{0} i_{1} \cdots i_{k}} & =\left\{x \in I: x \in I_{i_{0}}, f(x) \in I_{i_{1}}, \ldots, f^{k}(x) \in I_{i_{k}}\right\} \\
& =I_{i_{0}} \cap f^{-1}\left(I_{i_{1}}\right) \cap \cdots \cap f^{-k}\left(I_{i_{k}}\right) .
\end{aligned}
$$

In other words, $x \in I_{i_{0} i_{1} \cdots i_{k}}$ means that $\operatorname{ad}(x)=i_{0}, \operatorname{ad}(f(x))=i_{1}, \ldots, \operatorname{ad}\left(f^{k}(x)\right)=i_{k}$.

Consider the sign function $\varepsilon: \cup_{k=1}^{\infty} \mathcal{W}_{k} \rightarrow\{-1,0,1\}$ defined by

$$
\varepsilon\left(i_{1} \cdots i_{k}\right)=\prod_{j=1}^{k} \varepsilon(j)
$$


with $i_{1} \cdots i_{k} \in \mathcal{W}_{k}, \varepsilon\left(C_{j}\right)=0$ and $\varepsilon(j)=+1$ or $\varepsilon(j)=-1$, accordingly $f$ restricted to $I_{j}$ is increasing or decreasing.

The parity, with respect to the map $f$, of a given admissible word $i_{1}, \ldots, i_{k} \in \mathcal{W}_{k}$, is even if $\varepsilon\left(i_{1} \cdots i_{k}\right)=1$ and odd if $\varepsilon\left(i_{1} \cdots i_{k}\right)=-1$. From the order relation $1<C_{1} \prec 2 \prec \cdots \prec m+1$, inherited from the order of the intervals of the partition of the interval $I$, we introduce an order relation between symbolic sequences as follows: given any distinct sequences $\left(i_{k}\right)_{k \geq 1}$, $\left(j_{k}\right)_{k \geq 1} \in\left\{1, C_{1}, 2, \ldots, m+1\right\}^{\mathbb{N}_{0}}$, admitting that they have a common initial subsequence, that is, there is a natural $r \geq 0$ such that $i_{1} \cdots i_{r}=j_{1} \cdots j_{r}$ and $i_{r+1} \neq j_{r+1}$, we will say that $\left(i_{k}\right)_{k \geq 1} \prec$ $\left(j_{k}\right)_{k \geq 1}$ if and only if $i_{r+1} \prec j_{r+1}$ and $\varepsilon\left(i_{1} \cdots i_{r}\right)=1$ or $j_{r+1} \prec i_{r+1}$ and $\varepsilon\left(i_{1} \cdots i_{r}\right)=-1$.

\subsection{Symbolic Dynamics for the Infinite Dynamical System $(\mathcal{A}, T)$}

Now, consider an $m$-modal map $f$ in the class $C^{1}(I, I)$, for a certain interval $I \subset \mathbb{R}$, and the class of differentiable functions

$$
\mathscr{A}=\left\{\varphi \in C^{1}([0,1], I): \varphi^{\prime}(0)=\varphi^{\prime}(1)=0,|c p(\varphi)|<\infty\right\},
$$

where $|c p(\varphi)|$ denotes the number of critical points of $\varphi$.

Let $T$ be the operator

$$
\begin{aligned}
T: \mathcal{A} & \longrightarrow \mathcal{A}, \\
\varphi & \longmapsto f \circ \varphi .
\end{aligned}
$$

Note that this operator is well defined since $(f \circ \varphi)^{\prime}(0)=(f \circ \varphi)^{\prime}(1)=0$. Moreover, if $\phi \in \mathcal{A}$ and $\operatorname{Im}(\phi) \subset I$, then $\operatorname{Im}\left(T^{k} \phi\right) \subset I$ for every $k \in \mathbb{N}$. Therefore, we obtain a discrete dynamical system $(\mathcal{A}, T)$ in the sense that we have a set $\mathcal{A}$ (eventually with additional structure, a topology or a metric, for now not specified) and a self-map $T$, which characterizes the discrete time evolution.

In order to introduce a symbolic description for the discrete dynamical system $(\mathcal{A}, T)$, let us consider the decomposition of $\AA$ into the following classes (see [10-12]):

$$
\begin{gathered}
\mathcal{A}_{c}=\{\phi \in \mathcal{A}: \phi(x) \text { is constant in }[0,1]\}, \\
\mathcal{A}_{0}=\{\phi \in \mathcal{A}: \phi \text { has no critical points in }] 0,1[\}, \\
\mathcal{A}_{j}=\{\phi \in \mathcal{A}: \phi \text { has } j \text { critical points in }] 0,1[\}, j=1,2, \ldots .
\end{gathered}
$$

Let $\phi \in \mathcal{A}$ and let $\eta(\phi)$ be the number of nontrivial critical points of $\phi$ (inside [0,1]). In this case, if $\phi \in \mathcal{A}_{j}, j \in \mathbb{N}_{0}$, then $\eta(\phi)=j$ and the total number of critical points is $\eta(\phi)+2=$ $j+2$. We are interested in the symbolic description of the dynamical evolution of a function $\phi$ under iteration of $f$, which has essentially a topological meaning; therefore, the important aspect is to distinguish and codify the critical points and the critical values of $\phi$. Given $\phi \in \mathcal{A}$, we identify its critical points and collect the addresses and itineraries of the corresponding 
critical values. The generalized symbolic space is $\underline{\Sigma}:=\cup_{j \in \mathbb{N}_{0}} \Sigma^{j+1}$, where $\Sigma^{j+1}=\Sigma \times \Sigma \times \cdots \times \Sigma$ $(j+1$ times $)$, and we define the generalized address map for the space $A$ by

$$
\begin{aligned}
\underline{\mathrm{ad}}: & \mathcal{A} \\
\phi & \longmapsto \underline{\operatorname{ad}}(\phi):=\left(\operatorname{ad}\left(d_{0}\right), \operatorname{ad}\left(d_{1}\right), \ldots, \operatorname{ad}\left(d_{\eta(\phi)}\right), \operatorname{ad}\left(d_{\eta(\phi)+1}\right)\right)
\end{aligned}
$$

and the generalized itinerary map for the space $\mathcal{A}$ by

$$
\begin{aligned}
\text { it }: \mathcal{A} & \longrightarrow \underline{\Sigma} \\
\phi & \longmapsto \underline{\operatorname{it}}(\phi)=\left(\operatorname{it}\left(d_{0}\right), \operatorname{it}\left(d_{1}\right), \ldots, \operatorname{it}\left(d_{\eta(\phi)}\right), \operatorname{it}\left(d_{\eta(\phi)+1}\right)\right),
\end{aligned}
$$

where $d_{j}:=\phi\left(a_{j}\right), j=0,1, \ldots, \eta(\phi)+1$, are the critical values of $\phi$ in the interval $I$ (with $d_{0}=\phi(0)$ and $\left.d_{\eta(\phi)+1}=\phi(1)\right)$.

Let $i^{(j)}=i_{1}^{(j)} i_{2}^{(j)} \cdots$ be such that $i^{(j)}=\operatorname{it}\left(d_{j}\right), j=0,1,2, \ldots, \eta(\phi)+1$. The generalized shift map is then defined by

$$
\begin{aligned}
& \underline{\sigma}\left(i^{(0)}, \ldots, i^{(j)}, i^{(j+1)}, \ldots, i^{(\eta(\phi)+1)}\right) \\
& := \begin{cases}\left(\sigma\left(i^{(0)}\right), \ldots, \sigma\left(i^{(j)}\right), \sigma\left(i^{(j+1)}\right), \ldots, \sigma\left(i^{(\eta(\phi)+1)}\right)\right) & \\
\left(\sigma\left(i^{(0)}\right), \ldots, \sigma\left(i^{(j)}\right), \mathcal{K}_{i_{1}^{(j)}}, \ldots, \mathcal{K}_{i_{1}^{(j+1)}-1^{\prime}}\right. & \text { if } i_{1}^{(j)}=i_{1}^{(j+1)}, \\
\left.\sigma\left(i^{(j+1)}\right), \ldots, \sigma\left(i^{(\eta(\phi)+1)}\right)\right) & \text { if } i_{1}^{(j)} \neq i_{1}^{(j+1)}, i_{1}^{(j)} \prec i_{1}^{(j+1)}, \\
\left(\sigma\left(i^{(0)}\right), \ldots, \sigma\left(i^{(j)}\right), \mathcal{K}_{i_{1}^{(j)}-1}, \ldots, \mathcal{K}_{i_{1}^{(j+1)},}\right. & \\
\left.\sigma\left(i^{(j+1)}\right), \ldots, \sigma\left(i^{(\eta(\phi)+1)}\right)\right) & \text { if } i_{1}^{(j)} \neq i_{1}^{(j+1)}, i_{1}^{(j+1)}<i_{1}^{(j)},\end{cases}
\end{aligned}
$$

where $\mathcal{K}_{i_{1}^{(j)}}$ is the kneading sequence corresponding to the critical point of $f$, localized between the critical values $d_{j}$ and $d_{j+1}$, with $i_{1}^{(j)} \in\{1, \ldots, m+1\}, j=0,1,2, \ldots, \eta(\phi)$. We obtain a symbolic system $(\underline{\Sigma}, \underline{\sigma})$ associated to $(\mathscr{A}, T)$. Similarly to the finite-dimensional discrete dynamical systems, it is verified the following result.

Theorem 2.1. Let $\phi, \tilde{\phi} \in \mathcal{A}$ with $\phi \neq \tilde{\phi}$ so that $\underline{\operatorname{it}}(\phi)=\underline{\mathrm{it}}(\tilde{\phi})$, then

$$
\underline{\mathrm{it}}\left(T^{k} \phi\right)=\underline{\mathrm{it}}\left(T^{k} \tilde{\phi}\right), \quad k \in \mathbb{N}_{0} .
$$

Moreover,

$$
\underline{\sigma} \circ \underline{\text { it }}=\underline{\text { it }} \circ T .
$$

The previous definition and results allow us, knowing only the itineraries of the critical values of an initial condition $\phi_{0}$ and the kneading invariant of $f, \mathcal{K}_{f}$, to obtain explicitly the itineraries of the critical values of $\phi_{k}=T^{k} \phi_{0}, k \in \mathbb{N}$, see the next example. 
Example 2.2. Let us consider a 4-modal map $f$ characterized by the kneading invariant

$$
\mathcal{K}_{f}=\left(\left(555 C_{1}\right)^{\infty},\left(15 C_{2}\right)^{\infty},\left(53 C_{3}\right)^{\infty},\left(124 C_{4}\right)^{\infty}\right),
$$

(with the alphabet $\left\{1, C_{1}, 2, C_{2}, 3, C_{3}, 4, C_{4}, 5\right\}$ ), see Figure 1 . Now, we consider the generalized itinerary of the function $\phi_{0} \in \mathcal{A}$ given by $\underline{i t}\left(\phi_{0}\right)=\left((15)^{\infty},(253)^{\infty},(413)^{\infty}\right)$, with respect to the kneading invariant $\mathcal{K}_{f}$. Using the Theorem 2.1, we obtain the temporal evolution by $T$ of $\phi_{0}$ :

$$
\begin{aligned}
& \left((15)^{\infty},(253)^{\infty},(413)^{\infty}\right) \longrightarrow\left((51)^{\infty}, \mathcal{K}_{1},(532)^{\infty}, \mathcal{K}_{2}, \mathcal{K}_{3},(134)^{\infty}\right) \\
= & \left((51)^{\infty},\left(555 C_{1}\right)^{\infty},(532)^{\infty},\left(15 C_{2}\right)^{\infty},\left(53 C_{1}\right)^{\infty},(134)^{\infty}\right) \longrightarrow \\
& \left((15)^{\infty},\left(55 C_{1} 5\right)^{\infty},(325)^{\infty}, \mathcal{K}_{4}, \mathcal{K}_{3}, \mathcal{K}_{2}, \mathcal{K}_{1},\left(5 C_{2} 1\right)^{\infty},\left(3 C_{1} 5\right)^{\infty},(341)^{\infty}\right) \\
= & \left((15)^{\infty},\left(55 C_{1} 5\right)^{\infty},(325)^{\infty},\left(124 C_{4}\right)^{\infty},\left(53 C_{3}\right)^{\infty},\left(15 C_{2}\right)^{\infty},\left(555 C_{1}\right)^{\infty},\right. \\
& \left.\left(5 C_{2} 1\right)^{\infty},\left(3 C_{1} 5\right)^{\infty},(341)^{\infty}\right) \longrightarrow \\
& \left((51)^{\infty}, \mathcal{K}_{1}, \mathcal{K}_{2}, \mathcal{K}_{3}, \mathcal{K}_{4},\left(5 C_{1} 55\right)^{\infty}, \mathcal{K}_{4}, \mathcal{K}_{3},(253)^{\infty}, \mathcal{K}_{2}, \mathcal{K}_{1},\left(24 C_{4} 1\right)^{\infty}, \mathcal{K}_{1}, \mathcal{K}_{2}, \mathcal{K}_{3}, \mathcal{K}_{4},\right. \\
& \left.\left(3 C_{3} 5\right)^{\infty}, \mathcal{K}_{4}, \mathcal{K}_{3}, \mathcal{K}_{2}, \mathcal{K}_{1},\left(5 C_{2} 1\right)^{\infty}, \mathcal{K}_{1}, \mathcal{K}_{2}, \mathcal{K}_{3}, \mathcal{K}_{4},\left(55 C_{1} 5\right)^{\infty},\left(C_{2} 15\right)^{\infty},\left(C_{3} 53\right)^{\infty},(413)^{\infty}\right) \\
= & \left((51)^{\infty},\left(555 C_{1}\right)^{\infty},\left(15 C_{2}\right)^{\infty},\left(53 C_{3}\right)^{\infty},\left(124 C_{4}\right)^{\infty},\left(5 C_{1} 55\right)^{\infty},\left(124 C_{4}\right)^{\infty},\left(53 C_{3}\right)^{\infty},\right. \\
& (253)^{\infty},\left(15 C_{2}\right)^{\infty},\left(24 C_{4} 1\right)^{\infty},\left(555 C_{1}\right)^{\infty},\left(15 C_{2}\right)^{\infty},\left(53 C_{3}\right)^{\infty},\left(124 C_{4}\right)^{\infty},\left(3 C_{3} 5\right)^{\infty}, \\
& \left(124 C_{4}\right)^{\infty},\left(53 C_{3}\right)^{\infty},\left(15 C_{2}\right)^{\infty},\left(555 C_{1}\right)^{\infty},\left(5 C_{2} 1\right)^{\infty},\left(555 C_{1}\right)^{\infty},\left(15 C_{2}\right)^{\infty},\left(53 C_{3}\right)^{\infty}, \\
& \left.\left(124 C_{4}\right)^{\infty},\left(55 C_{1} 5\right)^{\infty},\left(C_{2} 15\right)^{\infty},\left(C_{3} 53\right)^{\infty},(413)^{\infty}\right) \longrightarrow \cdots
\end{aligned}
$$

\section{The Evolution and Distribution of the Aperiodic Critical Values of the Iterated Functions}

In our previous work, we analyze the evolution and distribution of the periodic critical values of the iterated functions when the kneading sequences of $f$ are periodic. Moreover, we developed the following algorithm based only on the kneading invariant of $f$. This algorithm computes symbolically all the itineraries of a sufficiently large number of critical values of iterated functions in few minutes. Using this algorithm, we overcome many difficulties in the study related with itineraries of critical values when we use numerical methods.

Algorithm 3.1 (to compute the itineraries of all critical values of $\phi_{n}$ ). Let $K_{1}, K_{2}, X_{0}$, and $n$ be the inputs and let $L$ and $Y$ be the outputs.

Step 0 . Set $L=\{\}, Z=X_{0}$ and $i=1$.

Step 1 . If $Y=\{\}$, compute $|Z|$ and append the sequence $\sigma(Z[1])$ to the vector $Y$. Set $j=1$ and go to the Step 2 .

Step 2 . If $j \leq|Z|-1$, then design by $y$ the first symbol of $Z[j]$ and by $z$ the first symbol of $Z[j+1]$, and go to the Step 3. Otherwise, go to the Step 7 .

Step 3. If $y=1$ (resp. $y=C_{2}$ or $y=2$ ) and $z=C_{2}$ or $z=2$ (resp. $z=1$ ), then append 


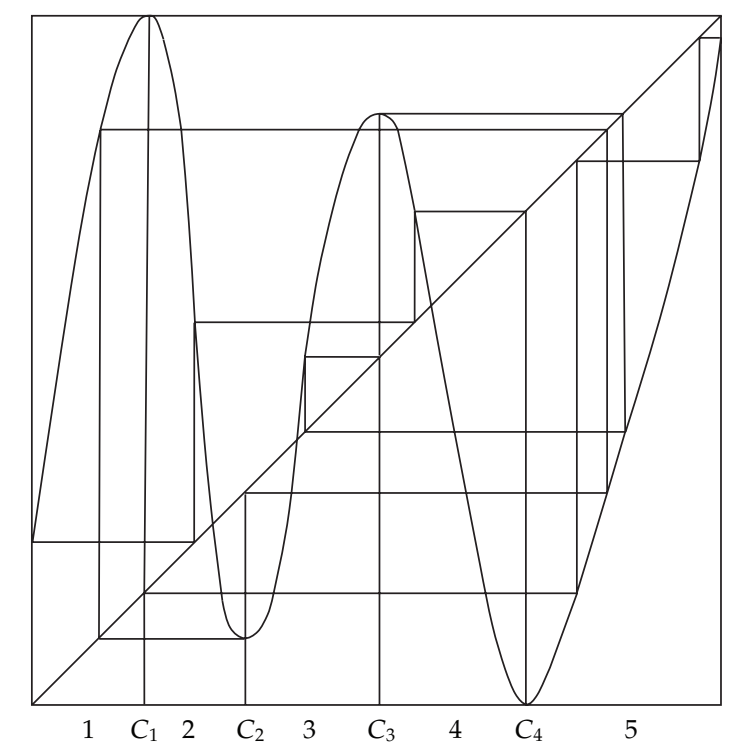

Figure 1: Graph of the map $f$ characterized by the kneading invariant $\mathcal{K}_{f}=\left(\left(555 C_{1}\right)^{\infty},\left(15 C_{2}\right)^{\infty}\right.$, $\left.\left(53 C_{3}\right)^{\infty},\left(124 C_{4}\right)^{\infty}\right)$.

$K_{1}$ to the vector $Y$ and the $\sigma(Z[j+1])$, set $j=j+1$ and return to the Step 2. Otherwise, go to the Step 4.

Step 4. If $y=3$ (resp. $y=C_{1}$ or $y=2$ ) and $z=C_{1}$, or $z=2$ (resp. $z=3$ ), then append $K_{2}$ to the vector $Y$ and the $\sigma(Z[j+1])$, set $j=j+1$, and return to the Step 2. Otherwise, go to the Step 5.

Step 5. If $y=1$ and $z=3$ then, append $K_{1}$ and $K_{2}$ to the vector $Y$ and the $\sigma(Z[j+1])$, set $j=j+1$, and return to the Step 2. Otherwise, go to the Step 6 .

Step 6. If $y=3$ and $z=1$, then append $K_{2}$ and the $K_{1}$ to the vector $Y$ and the $\sigma(Z[j+1])$, set $j=j+1$, and return to the Step 2 .

Step 7. Append the vector $Y$ to the set $L$ and set $Z=Y$. If $i=n$, the algorithm ends; otherwise, set $i=i+1$ and return to the Step 1 .

In the following example, we illustrate an implementation of the Algorithm 3.1 in Mathematica 6.0 for a bimodal $f$ with periodic kneading sequences.

Example 3.2. Let us consider a bimodal map $f: I \rightarrow I$ characterized by the kneading invariant $\mathcal{K}_{f}=\left(\left(332 C_{1}\right)^{\infty},\left(112 C_{2}\right)^{\infty}\right)$ and the function $\phi_{0} \in \mathcal{A}$ such that $\underline{\operatorname{it}}\left(\phi_{0}\right)=$ $\left((312)^{\infty},(32)^{\infty}\right)$. Considering

$$
K_{1}=332 C_{1}, \quad K_{2}=112 C_{2}, \quad X_{0}=\{312,32\}, \quad n=14,
$$


we obtain a large number of critical values of $\phi_{14}=T^{14} \phi_{0}$, in this case 181369 critical values for $\phi_{14}$. Moreover, we obtain the itineraries it $\left(\phi_{k}\right)$, for $k=0,1,2,3, \ldots, 7, \ldots, 14$, given by

$(123,23)$

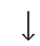

$\left(231,332 C_{1}, 32\right)$

$\downarrow$

$\left(312,112 C_{2}, 32 C_{1} 3,23\right)$

$\downarrow$

$\left(123,112 C_{2}, 332 C_{1}, 12 C_{2} 1,332 C_{1}, 112 C_{2}, 2 C_{1} 33,112 C_{2}, 32\right)$

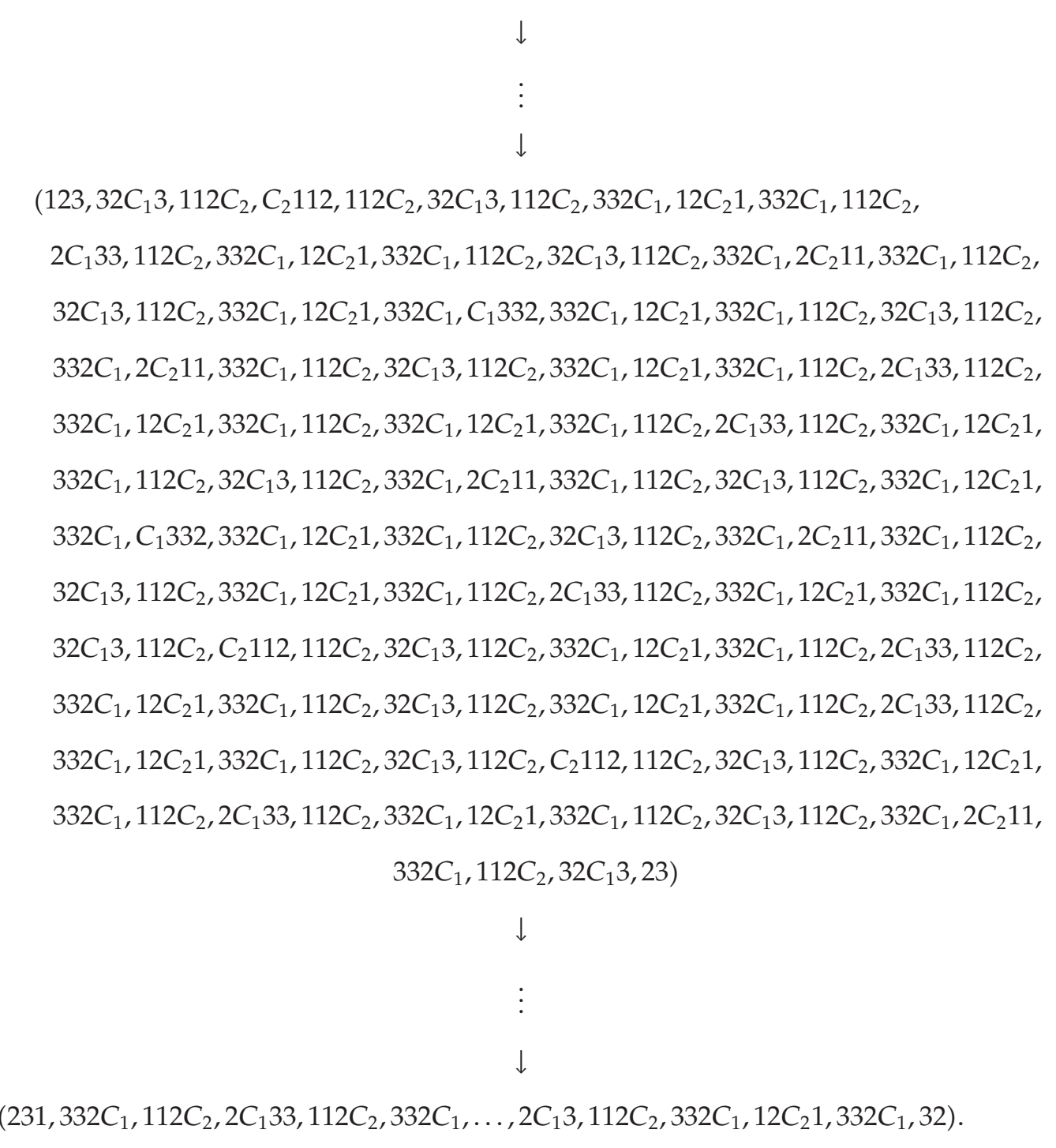


To apply the algorithm when the keading sequences of $f$ are periodic, we only consider the periodic part of each kneading sequence. However, there is no difficulty in applying the algorithm when the kneading sequences of $f$ are aperiodic. In this case, the length of the kneading sequences is not finite. Then, we make explicit at least the first $p$ symbols of each kneading sequence to compute the itineraries of critical values of $\phi_{p}=T^{p} \phi_{0}$, for some fixed $p \in \mathbb{N}$.

In this section, we analyze the evolution and distribution of the periodic critical values of the iterated functions when the kneading sequences of $f$ are aperiodic. As we present in the following results, the new distinct critical values obtained at each iteration depend on the kneading sequences of $f$. Then, the new critical values will also be aperiodic points which belong to some critical orbits. The number of different critical values will always grow and never stabilize. The reason is that the itinerary of each critical value will never repeat again under iteration. We also analyze the type of growth of these critical values.

The next result illustrates the set of the itineraries of all critical values of an iterated function $\phi_{k}$. As previously, $\eta\left(\phi_{0}\right)$ denotes the number of the nontrivial critical points of $\phi_{0}$ and $d_{j}, j=0,1,2, \ldots, \eta\left(\phi_{0}\right)+1$, denote the critical values of $\phi_{0}$. Let $v\left(\phi_{0}\right)$ be the number of the critical values of $\phi_{0}$.

Since the map $f$ restricted to a subinterval $I_{i}$ is monotone, $f$ is invertible if restricted to $I_{i}, i=1, \ldots, m$. Denote by $f_{i}^{-1}$ the inverse map $f_{\mid I_{i}}^{-1}: f\left(I_{i}\right) \rightarrow I_{i}$. Let $c_{i}, i=1, \ldots, m$, be the critical points of the map $f$, and let $U_{i} \subset I$ be the maximal interval containing $c_{i}$, consisting of points whose orbits tend to the stable periodic orbit of $c_{i}$, see [13].

Let $\Lambda_{i_{1} i_{2} \cdots i_{p} C_{i}} \subset I$ be given by $\Lambda_{i_{1} i_{2} \cdots i_{p} C_{i}}:=f_{i_{1}}^{-1} \circ f_{i_{2}}^{-1} \circ \cdots \circ f_{i_{p}}^{-1}\left(U_{i}\right), i=1, \ldots, m, i_{1} i_{2} \cdots i_{p} \in \mathcal{W}_{p}$, $p \in \mathbb{N}$. Therefore, the basin of attraction for $f$ is given by $\Lambda=\bigcup_{p=1}^{\infty} \bigcup_{i=1}^{m} \bigcup_{i_{1} i_{2} \cdots i_{p} \in \mathcal{V}_{p}} \Lambda_{i_{1} i_{2} \cdots i_{p} C_{i}}$.

Proposition 3.3. Let $f$ be an m-modal map, $\phi_{0} \in \mathcal{A}$ and $\phi_{k}=T^{k} \phi_{0}, k \in \mathbb{N}_{0}$. If the kneading sequences of $f, \mathcal{K}_{i}$, are aperiodic, then the itineraries of the critical values of $\phi_{k}, k \in \mathbb{N}_{0}$, will belong to the set

$$
\bigcup_{i=1}^{m}\left\{\sigma^{q}\left(\mathcal{K}_{i}\right), q=0,1,2, \ldots, k-1\right\} \cup \bigcup_{j=0}^{\eta\left(\phi_{0}\right)+1}\left\{\sigma^{l}\left(i t\left(d_{j}\right)\right), l=0,1,2, \ldots, k-1\right\},
$$

where $d_{j}, j=0,1,2, \ldots, \eta\left(\phi_{0}\right)+1$, are the critical values of $\phi_{0}$. Moreover, $v\left(\phi_{k}\right)$ will always grow and never stabilize.

Proof. Let $c_{i}, i=1,2, \ldots, m$, be the critical points of the map $f$. In [12], we proved that all critical points of $\phi_{k}=T^{k} \phi_{0}, k \in \mathbb{N}_{0}$ arise in the following two ways:

(i) a critical point of $\phi_{k-1}$ will be a critical point of $\phi_{k}$;

(ii) every critical point of $\phi_{k}$, which is not a critical point of $\phi_{k-1}$, is a point $y$ in which $\phi_{k-1}(y)=c_{i}$ for some $i=1,2, \ldots, m$.

Therefore, from (i), the critical values of $\phi_{k}$ correspond to the image under $f$ of the critical values of $\phi_{k-1}$.

From (ii), the new critical values of $\phi_{k}$ are always $f\left(c_{i}\right), i=1,2, \ldots, m$. Then the critical values of $\phi_{k}$ are either points in the set $\left\{f^{k}\left(d_{j}\right), k \in \mathbb{N}\right\}$ or points in the set 
$\bigcup_{i=1}^{m}\left\{f^{k}\left(c_{i}\right), k \in \mathbb{N}\right\}$. Recalling that $\operatorname{it}\left(f^{k}(x)\right)=\sigma^{k}(\operatorname{it}(x)), k \in \mathbb{N}_{0}$, for all $x \in I$, the itineraries of the critical values of $\phi_{k}$ are in the set

$$
\bigcup_{i=1}^{m}\left\{\sigma^{q}\left(\mathcal{K}_{i}\right), q=0,1,2, \ldots, k-1\right\} \cup \bigcup_{j=0}^{\eta\left(\phi_{0}\right)+1}\left\{\sigma^{l}\left(\operatorname{it}\left(d_{j}\right)\right), l=0,1,2, \ldots, k-1\right\} .
$$

Since we are considering aperiodic points $c_{i}, i=1,2, \ldots, m$, the set

$$
\bigcup_{i=1}^{m}\left\{f^{k}\left(c_{i}\right), k \in \mathbb{N}_{0}\right\}
$$

is infinite.

For a fixed $k \in \mathbb{N}_{0}$, we have that the number of distinct critical values of a certain $\phi_{k}$ is $v\left(\phi_{k}\right) \leq v\left(\phi_{k+1}\right)$. We have that $v\left(\phi_{k}\right)$ grows with $k$.

Let $i_{1} i_{2} \cdots$ be an aperiodic sequence in $\Sigma$ and let $N_{i_{1} i_{2} \ldots}(\phi)$ denote the number of times which $i_{1} i_{2} \cdots$ occurs as itinerary of a critical value of $\phi \in \mathcal{A}$. The following result illustrates the frequencies of occurence of the critical values whose itineraries are obtained of $\mathcal{K}_{i}, i=$ $1,2, \ldots, m$.

Proposition 3.4. Let $f$ be an m-modal map, $\phi_{0} \in \mathcal{A}$ (with $\phi_{0} \notin \mathcal{A}_{c}$ ), and $\phi_{k}=T^{k} \phi_{0}, k \in \mathbb{N}_{0}$. If the kneading sequences of $f, \mathcal{K}_{i}$, are aperiodic then for each $k \in \mathbb{N}_{0}$, we have

$$
N_{\sigma^{q}\left(\mathcal{K}_{i}\right)}\left(\phi_{k}\right) \leq N_{\sigma^{p}\left(\mathcal{K}_{i}\right)}\left(\phi_{k}\right),
$$

for $q>p$ with $p, q=0,1,2, \ldots, k-1, i=1,2, \ldots, m$.

Proof. As defined previously, $N_{\sigma^{q}\left(\mathcal{K}_{i}\right)}\left(\phi_{k}\right)$ is the number of times, which $\sigma^{q}\left(\mathcal{K}_{i}\right)$ occurs as itinerary of a critical value of $\phi_{k}$, for some fixed $i \in\{1,2, \ldots, m\}$ and some fixed $q \in$ $\{0,1,2, \ldots, k-1\}$.

If the itinerary of some critical values of $\phi_{k}$ is $\sigma^{q}\left(\mathcal{K}_{i}\right)$, then the itinerary of these critical values is $\mathcal{K}_{i}$ for $\phi_{k-q}$, which correspond to the new critical points of $\phi_{k-q}$. These new critical points are derived of the $(k-q-1)$ th-preimages of $c_{i}$, that is, $\phi_{k-q-1}(y)=c_{i}$.

Similarly, if the itinerary of some critical values of $\phi_{k}$ is $\sigma^{p}\left(\mathcal{K}_{i}\right)$, then these critical values correspond to new critical points of $\phi_{k-p}$ that are obtained of the $(k-p-1)$ th-preimages, under $f$ of $c_{i}$, that is, $\phi_{k-q-1}(y)=c_{i}$.

Since $q>p$, then $k-q-1<k-p-1$. In this case, the number of the admissible $k-1-q$-preimages is less or equal to the number of the admissible $k-1-p$-preimages since the number of laps of $f^{k-q}$ is less than the number of laps of $f^{k-p}$. Therefore,

$$
N_{\sigma^{q}\left(\mathcal{K}_{i}\right)}\left(\phi_{k}\right) \leq N_{\sigma^{p}\left(\mathcal{K}_{i}\right)}\left(\phi_{k}\right),
$$

for $q>p$ with $p, q=0,1,2, \ldots, k-1, i=1,2, \ldots, m$.

In order to illustrate the previous results, consider the following example. 
International Journal of Mathematics and Mathematical Sciences

Example 3.5. Consider the bimodal map $f$ characterized by the kneading invariant

$$
\mathcal{K}_{f}=(332311321321 \ldots, 113113323323 \ldots)
$$

which analytical expression is given approximately by

$$
f(x)=3.9333 x^{3}-0.00665413 x^{2}-2.9333 x+0.00665413
$$

and the function $\phi_{0} \in \mathcal{A}$ such that

$$
\underline{\operatorname{it}}\left(\phi_{0}\right)=\left(1^{\infty}, 3^{\infty}, 1^{\infty}, 3^{\infty}\right) \text {. }
$$

According to the Proposition 3.3, we have that the itineraries of the critical values of $\phi_{10}=T^{10} \phi_{0}$ belong to the set

$$
\begin{aligned}
&\left\{\sigma^{q}(332311321321 \ldots), q=0,1, \ldots, 9\right\} \cup\left\{\sigma^{q}(113113323323 \ldots), q=0,1, \ldots, 9\right\} \\
& \cup\left\{\sigma^{l}\left(1^{\infty}\right), l=0,1, \ldots, 9\right\} \cup\left\{\sigma^{l}\left(3^{\infty}\right), l=0,1, \ldots, 9\right\} \\
&=\{332311321321 \ldots, 32311321321 \ldots, 2311321321 \ldots, 311321321 \ldots, 11321321 \ldots, \\
& 1321321 \ldots, 321321 \ldots, 21321 \ldots, 1321 \ldots, 113113323323 \ldots, 13113323323 \ldots, \\
& 3113323323 \ldots, 113323323 \ldots, 13323323 \ldots, 3323323 \ldots, 323323 \ldots, 23323 \ldots \\
&\left.3323 \ldots, 323 \ldots, 1^{\infty}, 3^{\infty}\right\} .
\end{aligned}
$$

Regarding that $\mathcal{K}_{1}=332311321321 \ldots$ and $\mathcal{K}_{2}=113113323323 \ldots$, by the Proposition 3.4, we have

$$
\begin{aligned}
& N_{\sigma^{9}\left(\mathcal{K}_{1}\right)}\left(\phi_{10}\right) \leq N_{\sigma^{8}\left(\mathcal{K}_{1}\right)}\left(\phi_{10}\right) \leq N_{\sigma^{7}\left(\mathcal{K}_{1}\right)}\left(\phi_{10}\right) \leq \cdots \leq N_{\sigma\left(\mathcal{K}_{1}\right)}\left(\phi_{10}\right) \leq N_{\mathcal{K}_{1}}\left(\phi_{10}\right), \\
& N_{\sigma^{9}\left(\mathcal{K}_{2}\right)}\left(\phi_{10}\right) \leq N_{\sigma^{8}\left(\mathcal{K}_{2}\right)}\left(\phi_{10}\right) \leq N_{\sigma^{7}\left(\mathcal{K}_{2}\right)}\left(\phi_{10}\right) \leq \cdots \leq N_{\sigma\left(\mathcal{K}_{2}\right)}\left(\phi_{10}\right) \leq N_{\mathcal{K}_{2}}\left(\phi_{10}\right) .
\end{aligned}
$$


Indeed, we have

$$
\begin{array}{rll}
N_{\sigma^{9}\left(\mathcal{K}_{1}\right)}\left(\phi_{10}\right)=4, & N_{\sigma^{8}\left(\mathcal{K}_{1}\right)}\left(\phi_{10}\right)=12, & N_{\sigma^{7}\left(\mathcal{K}_{1}\right)}\left(\phi_{10}\right)=36, \\
N_{\sigma^{6}\left(\mathcal{K}_{1}\right)}\left(\phi_{10}\right)=100, & N_{\sigma^{5}\left(\mathcal{K}_{1}\right)}\left(\phi_{10}\right)=268, & N_{\sigma^{4}\left(\mathcal{K}_{1}\right)}\left(\phi_{10}\right)=716, \\
N_{\sigma^{3}\left(\mathcal{K}_{1}\right)}\left(\phi_{10}\right)=1908, & N_{\sigma^{2}\left(\mathcal{K}_{1}\right)}\left(\phi_{10}\right)=5060, & N_{\sigma\left(\mathcal{K}_{1}\right)}\left(\phi_{10}\right)=13412, \\
& N_{\mathcal{K}_{1}}\left(\phi_{10}\right)=35548, & \\
N_{\sigma^{9}\left(\mathcal{K}_{2}\right)}\left(\phi_{10}\right)=4, & N_{\sigma^{8}\left(\mathcal{K}_{2}\right)}\left(\phi_{10}\right)=12, & N_{\sigma^{7}\left(\mathcal{K}_{2}\right)}\left(\phi_{10}\right)=36, \\
N_{\sigma^{6}\left(\mathcal{K}_{2}\right)}\left(\phi_{10}\right)=92, & N_{\sigma^{5}\left(\mathcal{K}_{2}\right)}\left(\phi_{10}\right)=244, & N_{\sigma^{4}\left(\mathcal{K}_{2}\right)}\left(\phi_{10}\right)=644, \\
\mathrm{~N}_{\sigma^{3}\left(K_{2}\right)}\left(\phi_{10}\right)=1708, & \mathrm{~N}_{\sigma^{2}\left(\mathrm{~K}_{2}\right)}\left(\phi_{10}\right)=4524, & \mathrm{~N}_{\sigma\left(K_{2}\right)}\left(\phi_{10}\right)=11996, \\
& N_{\mathcal{K}_{2}}\left(\phi_{10}\right)=31780 . &
\end{array}
$$

Let us consider a bimodal map $f$ with aperiodic kneading sequences and growth number $s(f)$. Let $\phi_{0} \in \mathcal{A}$ be a function and $\phi_{k}=T^{k} \phi_{0}, k \in \mathbb{N}_{0}$. Recall that $N_{i^{(j)}}(\phi)$ is the number of times that $i^{(j)}, j=0,1,2, \ldots, v\left(\phi_{k}\right)$, occurs as itinerary of the critical values of $\phi \in \mathcal{A}$. We define the relative frequency of the critical values of $\phi_{k}$ by

$$
H_{i^{(j)}}\left(\phi_{k}\right):=\frac{N_{i^{(j)}}\left(\phi_{k}\right)}{\eta\left(\phi_{k}\right)+2}, \quad j=0,1,2, \ldots, v\left(\phi_{k}\right),
$$

where $i^{(j)}$ is the itinerary of the $j$-th critical value of $\phi_{k}$. These values $H_{i(j)}$ range between 0 and 1 and verify the following equality:

$$
H_{i^{(0)}}+H_{i^{(1)}}+\cdots+H_{i^{\left(v\left(\phi_{k}\right)\right)}}=1
$$

We denote by $H$ the set of the values $H_{i(j)}\left(\phi_{k}\right), j=0,1,2, \ldots, v\left(\phi_{k}\right)$, and we construct histograms using these values.

\section{Numerical Results}

Next, we present a numerical result that relates $H_{\sigma^{q}\left(\mathcal{K}_{i}\right)}\left(\phi_{k}\right)$, the relative frequencies of the critical values of $\phi_{k}$ given by $\sigma^{q}\left(\mathcal{K}_{i}\right), q=0,1,2, \ldots, k-1$, for each $i=1,2, \ldots, m$.

Let $\Lambda \subset I$ be the basin of attraction for $f$ and $\phi_{0} \in \mathcal{A}$ such that $\operatorname{Im}\left(\phi_{0}\right) \not \subset \Lambda$. If $\mathcal{K}_{i}$ is the kneading sequence of $f$, then

$$
\frac{H_{\sigma^{q}\left(\mathcal{K}_{i}\right)}\left(\phi_{k}\right)}{H_{\sigma^{q+n}\left(\mathcal{K}_{i}\right)}\left(\phi_{k}\right)} \longrightarrow s^{n}(f), \quad \text { since } k \longrightarrow \infty, q=0,1,2, \ldots, k-1, n=1,2, \ldots, k-2, i=1,2, \ldots, m \text {. }
$$

In a simple and concise way, we can explain the numerical result in (4.1), relating it with the growth rate of the critical points of $\phi_{k}$. There is a relationship between the growth 
rate of the critical points of $\phi_{k}$ and the growth number of $f, s(f)$. Since the growth rate of the critical points of $\phi_{k}$ is related with the growth of the preimages under $f$ of the critical points $c_{i}, i=1,2, \ldots, m$, we have that the growth of the number of each distinct critical value, that results of the kneading sequence $\mathcal{K}_{i}$, is related with growth of the critical points correspondent to the preimages under $f$ of the critical points $c_{i}$, for each $i \in\{1,2, \ldots, m\}$.

An estimation of the growth number of $f, s(f)$, is given by

$$
s(f) \approx \frac{\eta\left(\phi_{k}\right)+2}{\eta\left(\phi_{k-1}\right)+2}, \quad \text { as } k \longrightarrow \infty
$$

Usually, a practical way to compute the growth number of $f, s(f)$, when the kneading sequences of $f$ are periodic is through the spectral radius of the transition matrix associated to the kneading invariant of $f$, see [14]. Using the algorithm, we spend less time computing the growth number of $f$ than computing the transition matrix associated to the kneading invariant of $f$. Here, we compute numerically the total number of critical points of the iterated functions calculating the total number of the itineraries of their critical values.

In the following example, we illustrate the previous result.

Example 4.1. Consider the bimodal map $f: I \rightarrow I$ characterized by the kneading invariant

$$
\mathcal{K}_{f}=(331233113212 \ldots, 112223213311 \ldots),
$$

whose analytical expression is given approximately by

$$
f(x)=3.91322 x^{3}+0.0232761 x^{2}-2.91322 x-0.0232761 .
$$

Let $\phi_{0} \in \mathcal{A}$ be a function such that

$$
\underline{\text { it }}\left(\phi_{0}\right)=\left(1^{\infty}, 3^{\infty}\right),
$$

whose analytical expression is given by $\phi_{0}(x)=-\cos (\pi x)$.

An estimation of the growth number of $f, s(f)$, is given by

$$
s(f) \approx \frac{\eta\left(\phi_{k}\right)+2}{\eta\left(\phi_{k-1}\right)+2}=\frac{170724}{65482}=2.60719 \ldots, \quad \text { as } k \longrightarrow \infty .
$$

The number $\eta\left(\phi_{k}\right)$ is easily computed symbolically using the algorithm. The itineraries of the critical values correspondent to the new critical points of $\phi_{12}=T^{12} \phi_{0}$ are given by $\sigma^{q}\left(\mathcal{K}_{i}\right), q=0,1,2, \ldots, 11, i=1,2$, and are presented in the following set ordered by increasing $k$, which is suitable to present graphically

$$
\left\{\mathcal{K}_{2}, \sigma\left(\mathcal{K}_{2}\right), \sigma^{2}\left(\mathcal{K}_{2}\right), \sigma^{3}\left(\mathcal{K}_{2}\right), \ldots, \sigma^{11}\left(\mathcal{K}_{2}\right), \mathcal{K}_{1}, \sigma\left(\mathcal{K}_{1}\right), \sigma^{2}\left(\mathcal{K}_{1}\right), \sigma^{3}\left(\mathcal{K}_{1}\right), \ldots, \sigma^{11}\left(\mathcal{K}_{1}\right)\right\} .
$$


The respective values $N_{\sigma^{q}\left(\mathcal{K}_{i}\right)}\left(\phi_{12}\right), q=0,1,2, \ldots, 11, i=1,2$, are

$\{53765,20621,7911,3035,1163,447,171,65,25,9,3,1,51477,19749$,

$7573,2905,1113,427,163,63,23,9,3,1\}$.

The relative frequencies of the critical values of $\phi_{12}$ whose itineraries are $\sigma^{q}\left(\mathcal{K}_{i}\right)$,

$$
H_{\sigma^{q}\left(\mathcal{K}_{i}\right)}\left(\phi_{12}\right)=\frac{N_{\sigma^{q}\left(\mathcal{K}_{i}\right)}\left(\phi_{12}\right)}{\eta\left(\phi_{12}\right)+2}
$$

with $\eta\left(\phi_{12}\right)=170444, q=0,1, \ldots, 11, i=1,2$, are given in the set

$$
\begin{aligned}
& H=\{0.314931 \ldots, 0.120788 \ldots, 0.046339 \ldots, 0.0177776 \ldots, 0.00681232 \ldots, \\
& 0.00261832 \ldots, 0.00100164 \ldots, 0.00038074 \ldots, 0.000146439 \ldots, 0.000052717, \ldots, \\
& 0.0000175726 \ldots, 0.301529 \ldots, 0.115681 \ldots, 0.0443592 \ldots, 0.0170162 \ldots, \\
& 0.00651945 \ldots, 0.00250117 \ldots, 0.00095478 \ldots, 0.000369025 \ldots, 0.000134724 \ldots, \\
&0.0000527179 \ldots, 0.0000175726 \ldots\}
\end{aligned}
$$

These values (dependent only on the kneading invariant of $f$ ) are independent of the initial functions and are presented in the histogram of Figure 2. Note that in this histogram, the distribution of the critical values is not symmetric and has two peaks at $\mathcal{K}_{1}$ and $\mathcal{K}_{2}$. In this example, the kneading sequences $\mathcal{K}_{1}$ and $\mathcal{K}_{2}$ are not symmetric, and we have

$$
H_{\sigma^{q}\left(\mathcal{K}_{1}\right)}\left(\phi_{k}\right) \leq H_{\sigma^{q}\left(\mathcal{K}_{2}\right)}\left(\phi_{k}\right), \quad \text { as } k \longrightarrow \infty, q=0,1, \ldots, 11 \text {. }
$$

In general, if

$$
H_{\mathcal{K}_{1}}\left(\phi_{k}\right) \leq H_{\mathcal{K}_{2}}\left(\phi_{k}\right)
$$

then

$$
H_{\sigma^{q}\left(\mathcal{K}_{1}\right)}\left(\phi_{k}\right) \leq H_{\sigma^{q}\left(\mathcal{K}_{2}\right)}\left(\phi_{k}\right), \quad \text { as } k \longrightarrow \infty
$$

In this particular case, according to the values $H_{\sigma^{q}\left(\mathcal{K}_{i}\right)}\left(\phi_{12}\right)$, we have

$$
H_{\mathcal{K}_{1}} \leq H_{\mathcal{K}_{2}} \leq H_{\sigma\left(\mathcal{K}_{1}\right)} \leq H_{\sigma\left(\mathcal{K}_{2}\right)} \leq \cdots \leq H_{\sigma^{11}\left(\mathcal{K}_{1}\right)} \leq H_{\sigma^{11}\left(\mathcal{K}_{2}\right)} .
$$




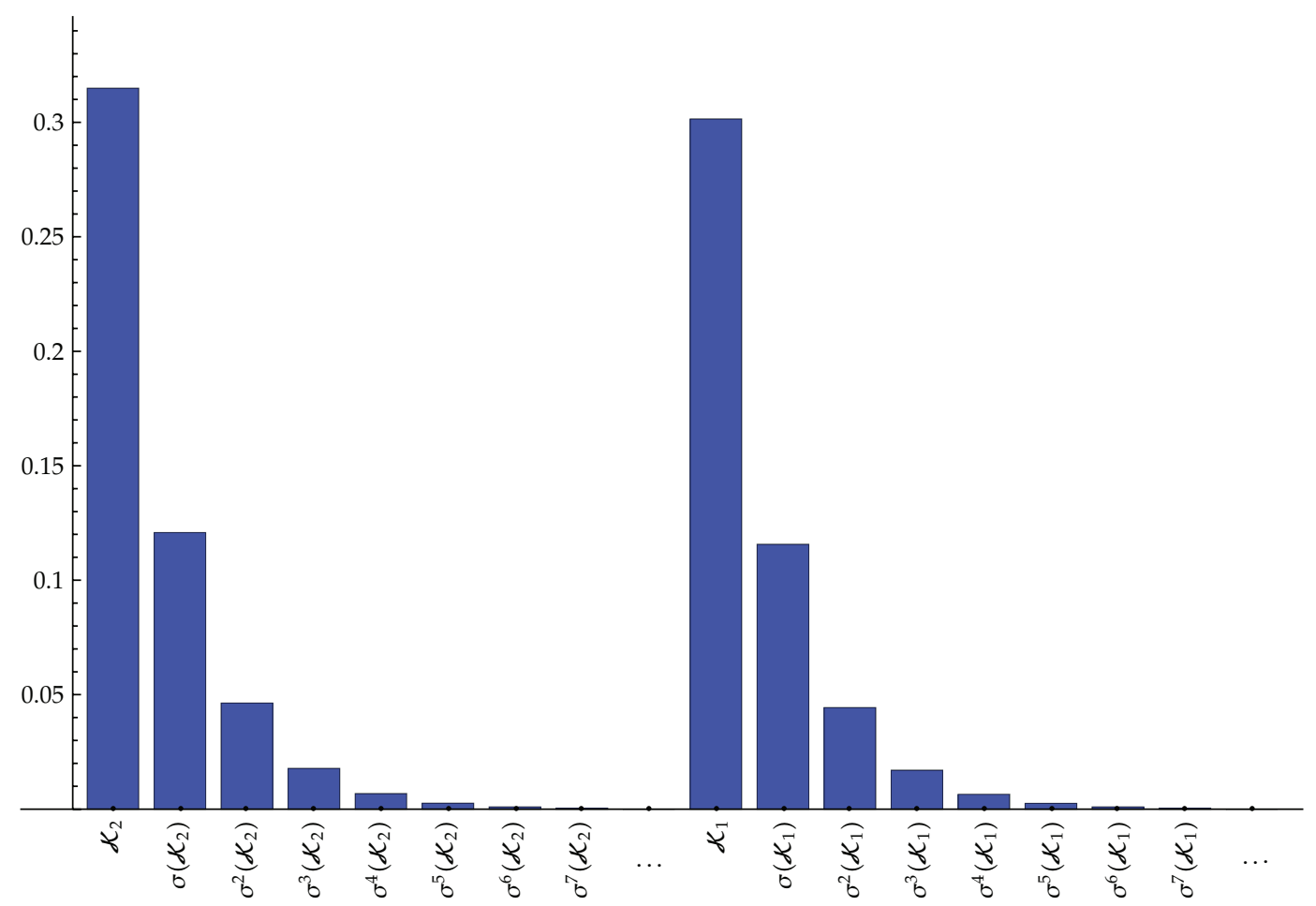

Figure 2: Histogram of the relative frequencies of the distinct critical values of $\phi_{12}=T^{12} \phi_{0}$, whose itineraries are given by $\sigma^{q}\left(\mathcal{K}_{1}\right), \sigma^{q}\left(\mathcal{K}_{2}\right), q=0,1,2,3, \ldots$ Note that the order of these itineraries is $\mathcal{K}_{2}$, $\sigma\left(\mathcal{K}_{2}\right), \sigma^{2}\left(\mathcal{K}_{2}\right), \sigma^{3}\left(\mathcal{K}_{2}\right), \ldots, \mathcal{K}_{1}, \sigma\left(\mathcal{K}_{1}\right), \sigma^{2}\left(\mathcal{K}_{1}\right), \sigma^{3}\left(\mathcal{K}_{1}\right), \ldots$

Moreover, we have the following:

$$
\begin{array}{llll}
\frac{H_{\mathcal{K}_{1}}\left(\phi_{k}\right)}{H_{\sigma\left(\mathcal{K}_{1}\right)}\left(\phi_{k}\right)}=2.60656 \ldots, & \frac{H_{\sigma\left(\mathcal{K}_{1}\right)}\left(\phi_{k}\right)}{H_{\sigma^{2}\left(\mathcal{K}_{1}\right)}\left(\phi_{k}\right)}=2.60782 \ldots, & \frac{H_{\sigma^{2}\left(\mathcal{K}_{1}\right)}\left(\phi_{k}\right)}{H_{\sigma^{3}\left(\mathcal{K}_{1}\right)}\left(\phi_{k}\right)}=2.60688, \ldots, \\
\frac{H_{\sigma^{3}\left(\mathcal{K}_{1}\right)}\left(\phi_{k}\right)}{H_{\sigma^{4}}\left(\mathcal{K}_{1}\right)}\left(\phi_{k}\right) & =2.61006 \ldots, & \frac{H_{\sigma^{4}\left(\mathcal{K}_{1}\right)}\left(\phi_{k}\right)}{H_{\sigma^{5}\left(\mathcal{K}_{1}\right)}\left(\phi_{k}\right)}=2.60656 \ldots, & \frac{H_{\sigma^{5}\left(\mathcal{K}_{1}\right)}\left(\phi_{k}\right)}{H_{\sigma^{6}\left(\mathcal{K}_{1}\right)}\left(\phi_{k}\right)}=2.61963, \ldots, \\
\frac{H_{\mathcal{K}_{1}}\left(\phi_{k}\right)}{H_{\sigma^{2}\left(\mathcal{K}_{1}\right)}\left(\phi_{k}\right)}=6.7944 \ldots, & \frac{H_{\sigma\left(\mathcal{K}_{1}\right)}\left(\phi_{k}\right)}{H_{\sigma^{3}\left(\mathcal{K}_{1}\right)}\left(\phi_{k}\right)}=6.79828 \ldots, & \frac{H_{\sigma^{2}\left(\mathcal{K}_{1}\right)}\left(\phi_{k}\right)}{H_{\sigma^{4}\left(\mathcal{K}_{1}\right)}\left(\phi_{k}\right)}=6.80413, \ldots, \\
\frac{H_{\sigma^{3}\left(\mathcal{K}_{1}\right)}\left(\phi_{k}\right)}{H_{\sigma^{5}\left(\mathcal{K}_{1}\right)}\left(\phi_{k}\right)}=6.80328 \ldots, & \frac{H_{\sigma^{4}\left(\mathcal{K}_{1}\right)}\left(\phi_{k}\right)}{H_{\sigma^{6}\left(\mathcal{K}_{1}\right)}\left(\phi_{k}\right)}=6.82822 \ldots, & \frac{H_{\sigma^{5}\left(\mathcal{K}_{1}\right)}\left(\phi_{k}\right)}{H_{\sigma^{7}\left(\mathcal{K}_{1}\right)}\left(\phi_{k}\right)}=6.77778, \ldots, \\
\frac{H_{\mathcal{K}_{1}}\left(\phi_{k}\right)}{H_{\sigma^{3}\left(\mathcal{K}_{1}\right)}\left(\phi_{k}\right)}=17.7201 \ldots, & \frac{H_{\sigma\left(\mathcal{K}_{1}\right)}\left(\phi_{k}\right)}{H_{\sigma^{4}\left(\mathcal{K}_{1}\right)}\left(\phi_{k}\right)}=17.7439 \ldots, & \frac{H_{\sigma^{2}\left(\mathcal{K}_{1}\right)}\left(\phi_{k}\right)}{H_{\sigma^{5}\left(\mathcal{K}_{1}\right)}\left(\phi_{k}\right)}=17.7354, \ldots, \\
\frac{H_{\mathcal{K}_{1}}\left(\phi_{k}\right)}{H_{\sigma^{4}\left(\mathcal{K}_{1}\right)}\left(\phi_{k}\right)}=46.2507 \ldots, & \frac{H_{\sigma\left(\mathcal{K}_{1}\right)}\left(\phi_{k}\right)}{H_{\sigma^{5}\left(\mathcal{K}_{1}\right)}\left(\phi_{k}\right)}=46.2506 \ldots, & \frac{H_{\sigma^{2}\left(\mathcal{K}_{1}\right)}\left(\phi_{k}\right)}{H_{\sigma^{6}\left(\mathcal{K}_{1}\right)}\left(\phi_{k}\right)}=46.4601, \ldots,
\end{array}
$$




$$
\begin{array}{llll}
\frac{H_{\mathcal{K}_{2}}\left(\phi_{k}\right)}{H_{\sigma\left(\mathcal{K}_{2}\right)}\left(\phi_{k}\right)}=2.60729 \ldots, & \frac{H_{\sigma\left(\mathcal{K}_{2}\right)}\left(\phi_{k}\right)}{H_{\sigma^{2}\left(\mathcal{K}_{2}\right)}\left(\phi_{k}\right)}=2.60662 \ldots, & \frac{H_{\sigma^{2}\left(\mathcal{K}_{2}\right)}\left(\phi_{k}\right)}{H_{\sigma^{3}\left(\mathcal{K}_{2}\right)}\left(\phi_{k}\right)}=2.60659, \ldots, \\
\frac{H_{\sigma^{3}\left(\mathcal{K}_{2}\right)}\left(\phi_{k}\right)}{H_{\sigma^{4}\left(\mathcal{K}_{2}\right)}\left(\phi_{k}\right)}=2.60963 \ldots, & \frac{H_{\sigma^{4}\left(\mathcal{K}_{2}\right)}\left(\phi_{k}\right)}{H_{\sigma^{5}\left(\mathcal{K}_{2}\right)}\left(\phi_{k}\right)}=2.60179 \ldots, & \frac{H_{\sigma^{5}\left(\mathcal{K}_{2}\right)}\left(\phi_{k}\right)}{H_{\sigma^{6}\left(\mathcal{K}_{2}\right)}\left(\phi_{k}\right)}=2.61404, \ldots, \\
\frac{H_{\mathcal{K}_{2}}\left(\phi_{k}\right)}{H_{\sigma^{2}\left(\mathcal{K}_{2}\right)}\left(\phi_{k}\right)}=6.79623 \ldots, & \frac{H_{\sigma\left(\mathcal{K}_{2}\right)}\left(\phi_{k}\right)}{H_{\sigma^{3}\left(\mathcal{K}_{2}\right)}\left(\phi_{k}\right)}=6.7944 \ldots, & \frac{H_{\sigma^{2}\left(\mathcal{K}_{2}\right)}\left(\phi_{k}\right)}{H_{\sigma^{4}\left(\mathcal{K}_{2}\right)}\left(\phi_{k}\right)}=6.80224, \ldots, \\
\frac{H_{\sigma^{3}\left(\mathcal{K}_{2}\right)}\left(\phi_{k}\right)}{H_{\sigma^{5}\left(\mathcal{K}_{2}\right)}\left(\phi_{k}\right)}=6.78971 \ldots, & \frac{H_{\sigma^{4}\left(\mathcal{K}_{2}\right)}\left(\phi_{k}\right)}{H_{\sigma^{6}\left(\mathcal{K}_{2}\right)}\left(\phi_{k}\right)}=6.80117 \ldots, & \frac{H_{\sigma^{5}\left(\mathcal{K}_{2}\right)}\left(\phi_{k}\right)}{H_{\sigma^{7}\left(\mathcal{K}_{2}\right)}\left(\phi_{k}\right)}=6.87692, \ldots, \\
\frac{H_{\mathcal{K}_{2}}\left(\phi_{k}\right)}{H_{\sigma^{3}\left(\mathcal{K}_{2}\right)}\left(\phi_{k}\right)}=17.715 \ldots, & \frac{H_{\sigma\left(\mathcal{K}_{2}\right)}\left(\phi_{k}\right)}{H_{\sigma^{4}\left(\mathcal{K}_{2}\right)}\left(\phi_{k}\right)}=17.7309 \ldots, & \frac{H_{\sigma^{2}\left(\mathcal{K}_{2}\right)}\left(\phi_{k}\right)}{H_{\sigma^{5}\left(\mathcal{K}_{2}\right)}\left(\phi_{k}\right)}=17.698, \ldots, \\
\frac{H_{\mathcal{K}_{2}}\left(\phi_{k}\right)}{H_{\sigma^{4}\left(\mathcal{K}_{2}\right)}\left(\phi_{k}\right)}=46.2296 \ldots, & \frac{H_{\sigma\left(\mathcal{K}_{2}\right)}\left(\phi_{k}\right)}{H_{\sigma^{5}\left(\mathcal{K}_{2}\right)}\left(\phi_{k}\right)}=46.132 \ldots, & \frac{H_{\sigma^{2}\left(\mathcal{K}_{2}\right)}\left(\phi_{k}\right)}{H_{\sigma^{6}\left(\mathcal{K}_{2}\right)}\left(\phi_{k}\right)}=46.2632 \ldots,
\end{array}
$$

where $s(f)=2.60719 \ldots,(s(f))^{2}=6.79744 \ldots,(s(f))^{3}=17.7222 \ldots$, and $(s(f))^{4}=46.2052 \ldots$. In general, we have

$$
\frac{H_{\sigma^{q}\left(\mathcal{K}_{i}\right)}\left(\phi_{k}\right)}{H_{\sigma^{q+n}\left(\mathcal{K}_{i}\right)}\left(\phi_{k}\right)} \longrightarrow s^{n}(f), \quad \text { as } k \longrightarrow \infty, q=0,1,2, \ldots, k-1, n=1,2, \ldots, k-2, i=1,2
$$

\section{Acknowledgment}

M. F. Correia was supported by Calouste Gulbenkian Foundation. This work has been partially supported by the research center CIMA-UE, FCT-Portugal funding program.

\section{References}

[1] E. Y. Romanenko and A. N. Sharkovsky, "From boundary value problems to difference equations: a method of investigation of chaotic vibrations," International Journal of Bifurcation and Chaos in Applied Sciences and Engineering, vol. 9, no. 7, pp. 1285-1306, 1999.

[2] A. N. Sharkovsky, Y. Maǐstrenko, and E. Y. Romanenko, Difference equations and their applications, vol. 250, Kluwer Academic, 1993.

[3] A. N. Sharkovsky, "Difference equations and boundary value problems," in Proceedings of the 6th International Conference on Difference Equations (ICDEA '01), New Progress in Difference Equations, pp. 3-22, Taylor \& Francis, 2003.

[4] R. Severino, A. N. Sharkovsky, J. S. Ramos, and S. Vinagre, "Symbolic dynamics in boundary value problems," Grazer Mathematische Berichte, vol. 346, pp. 393-402, 2004.

[5] R. Severino, A. Sharkovsky, J. S. Ramos, and S. Vinagre, "Topological invariants in a model of a timedelayed Chua's circuit," Nonlinear Dynamics, vol. 44, no. 1-4, pp. 81-90, 2006.

[6] S. Vinagre, R. Severino, and J. S. Ramos, "Topological invariants in nonlinear boundary value problems," Chaos, Solitons E Fractals, vol. 25, no. 1, pp. 65-78, 2005. 
[7] M. F. Correia, C. C. Ramos, and S. Vinagre, "Evolution and distribution of the periodic critical values of iterated dierentiable functions". In press.

[8] J. Milnor and W. Thurston, "On iterated maps of the interval," in Lecture Notes in Mathematics, J. C. Alexander, Ed., vol. 1342, pp. 465-563, Springer, Berlin, Germany, 1988.

[9] M. Misiurewicz and W. Szlenk, "Entropy of piecewise monotone mappings," Studia Mathematica, vol. 67, no. 1, pp. 45-63, 1980.

[10] M. F. Correia, C. C. Ramos, and S. Vinagre, "Symbolic dynamics for iterated smooth functions," in Grazer Mathematische Berichte, vol. 354, pp. 26-36, 2009.

[11] M. F. Correia, C. C. Ramos, and S. Vinagre, "On the iteration of smooth maps," in Discrete Dynamics and Difference Equations: Proceedings of the Twelfth International Conference on Difference Equations and Applications, pp. 223-230, World Scientific Publishing, 2010.

[12] M. F. Correia, C. C. Ramos, and S. M. Vinagre, "Iteration of differentiable functions under $m$-modal maps," International Journal of Pure and Applied Mathematics, vol. 70, no. 2, pp. 151-166, 2011.

[13] J. Guckenheimer, "Sensitive dependence to initial conditions for one-dimensional maps," Communications in Mathematical Physics, vol. 70, no. 2, pp. 133-160, 1979.

[14] R. Severino, Invariantes Topológicos e Algébricos em Sistemas Dinâmicos Discretos [Ph.D. thesis], Instituto Superior Técnico, 2000. 


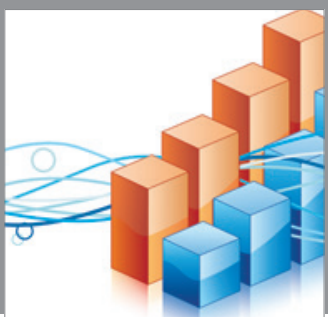

Advances in

Operations Research

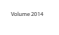

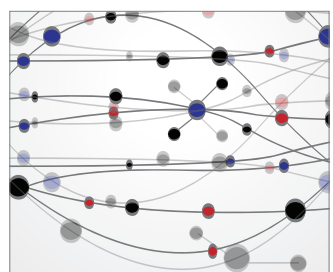

\section{The Scientific} World Journal
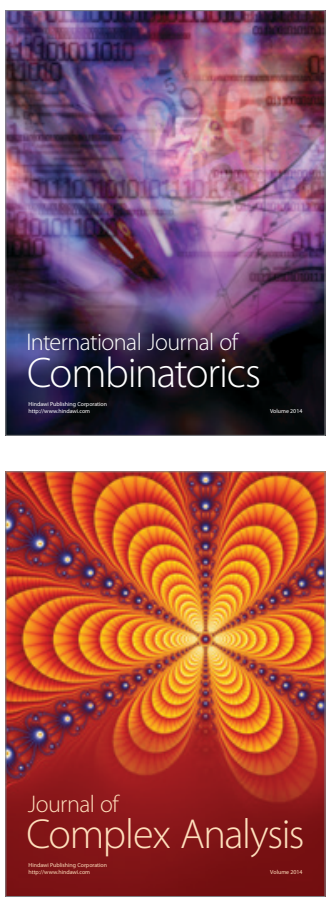

International Journal of

Mathematics and

Mathematical

Sciences
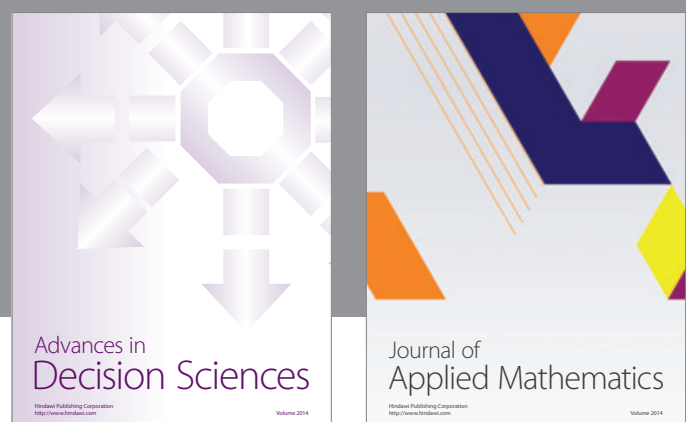

Journal of

Applied Mathematics
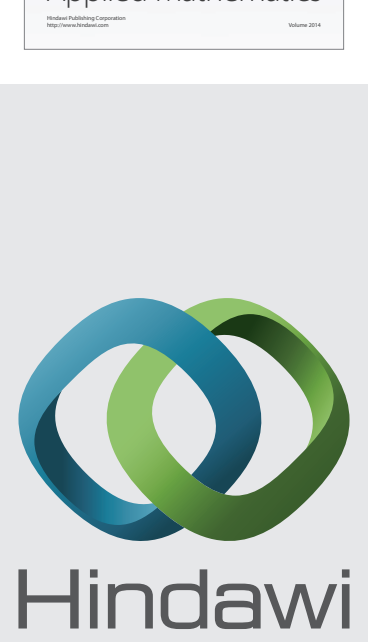

Submit your manuscripts at http://www.hindawi.com
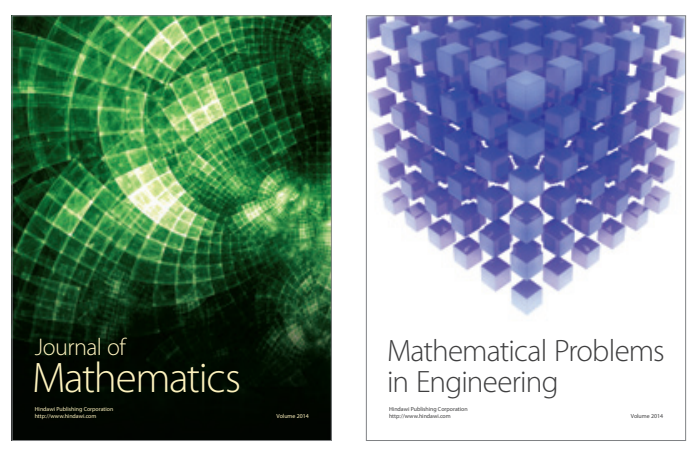

Mathematical Problems in Engineering
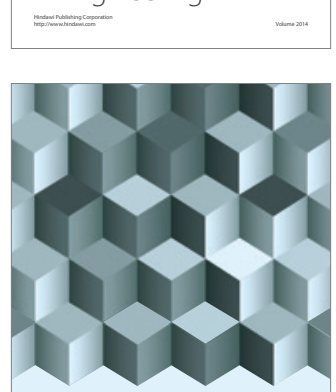

Journal of

Function Spaces
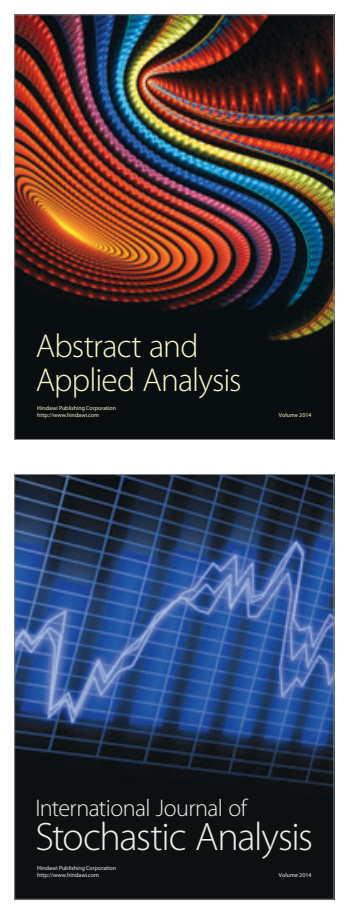

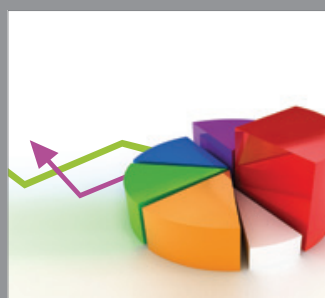

ournal of

Probability and Statistics

Promensencen
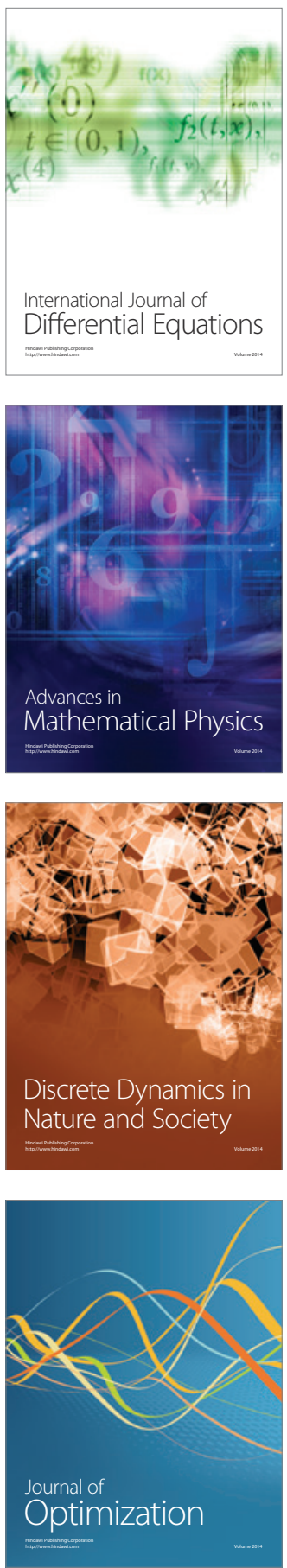